\author{
Giorgio Feliciani \\ (ordinario di Diritto canonico nella Facoltà di Giurisprudenza \\ dell'Università Cattolica del Sacro Cuore di Milano)
}

\title{
La laicità dello Stato negli insegnamenti di Benedetto XVI *
}

SOMMARIO: 1. Da Giovanni Paolo II a Benedetto XVI - 2. Il principio di laicità 3. Laicità e libertà - 4. Le degenerazioni del principio - 5. Laicità sana e laicità positiva - 6. Chiesa e Stato - 7. Riflessioni conclusive.

\section{1 - Da Giovanni Paolo II a Benedetto XVI}

Negli insegnamenti di Giovanni Paolo II la libertà religiosa occupa un posto di singolare rilievo, persino sotto il profilo quantitativo. Infatti una raccolta dei suoi pronunciamenti in materia nei primi venti anni di pontificato annovera dieci documenti di notevole importanza e più di quattrocento menzioni per così dire occasionali ${ }^{1}$. Una tale insistenza non deve sorprendere: il pontefice polacco riconosceva nella libertà religiosa un diritto che, stando "alla radice di ogni altro diritto e di ogni altra libertà"2, può senz'altro essere considerato "uno dei pilastri che sorreggono l'edificio dei diritti umani" 30 , ancor più precisamente, la sua "pietra angolare" 4 . Un diritto che "esiste in ogni persona ed esiste sempre, anche nell'ipotesi che non venga esercitato o sia violato dagli stessi soggetti cui inerisce ${ }^{\prime \prime}$.

\footnotetext{
* Contributo destinato agli Studi in onore di Rinaldo Bertolino
}

1 Università Cattolica del Sacro Cuore, Centro di ricerche per lo studio della dottrina sociale della Chiesa, La libertà religiosa negli insegnamenti di Giovanni Paolo II (1978-1998), Milano, Vita e pensiero, 2000.

2 Discorso ai partecipanti al IX Colloquio internazionale romanistico canonistico organizzato dalla Pontificia Università Lateranense, 11 dicembre 1993, n. 3 (d'ora innanzi Discorso al IX Colloquio). Salvo diversa indicazione tutti gli atti pontifici citati nel presente studio sono consultabili sul sito www.vatican.va.

3 Discorso ai membri della "Società Paasikivi" nella sala dei concerti della "Finlandia Hall", 5 giugno 1989, n. 2.

${ }^{4}$ Messaggio per la celebrazione della XXIV giornata della Pace, 8 dicembre 1990, $\mathrm{n}$. 5 .

${ }^{5}$ Discorso ai partecipanti al V Colloquio internazionale di studi giuridici, 10 marzo 1984, n. 5. Per più ampie notizie vedi G. FELICIANI, La libertà religiosa nel magistero di Giovanni Paolo II, in Rivista internazionale dei diritti dell'uomo, 12 (1999), pp. 158-167. 
Benedetto XVI si colloca nel solco di questi insegnamenti - come dimostra da ultimo e in modo particolarmente evidente il Messaggio del $1^{\circ}$ gennaio 2011, intitolato "Libertà religiosa, via per la pace"6 - ma con una certa novità di accenti e di sottolineature che appare dovuta sia alla sua formazione e allo stesso suo temperamento, sia alle circostanze a cui si è trovato e si trova a fare fronte.

\section{2 - Il principio di laicità}

In questa prospettiva merita soffermarsi sulla specifica attenzione dedicata da Benedetto XVI alla complessa problematica concernente la laicità dello Stato. A suo giudizio essa, in quanto consistente nella distinzione tra i due poteri", "non è in contrasto con il messaggio cristiano, ma piuttosto è ad esso debitrice" 8 . Infatti "la distinzione tra religione e politica è un ottenimento proprio del Cristianesimo e uno dei suoi fondamentali contributi storici e culturali"9. Ancor più precisamente e diffusamente, nella enciclica "Deus caritas est", il pontefice mette in luce come

"la distinzione tra ciò che è di Cesare e ciò che è di Dio, cioè la distinzione tra Stato e Chiesa, o, come dice il Vaticano II, l'autonomia delle realtà temporali" appartenga "alla struttura fondamentale del Cristianesimo" 10 .

Affermazioni indubbiamente significative, ma che per lo più si limitano a riecheggiare il magistero precedente. Già Pio XII riconduceva la laicità dello Stato a quella distinzione dei poteri che costituisce "uno dei principi della dottrina cattolica"11, un insegnamento citato e confermato

${ }^{6}$ Messaggio per la celebrazione della XLIV giornata mondiale della pace, $1^{\circ}$ gennaio 2011 (d'ora innanzi Messaggio, $1^{\circ}$ gennaio 2011). Si avverta che il presente studio prende in esame gli interventi di Benedetto XVI fino a tutto il gennaio 2011.

7 "le principe de laïcité consiste en une saine distinction des pouvoirs", Discours à S.E. Bernard Kessedjian ambassadeur de France près du Saint-Siège à l'occasion de la présentation des lettres de créance, 19 dicembre 2005 (d'ora innanzi Discours à l'ambassadeur de France, 19 dicembre 2005).

${ }^{8}$ Lettera all'onorevole Pier Ferdinando Casini Presidente della Camera dei Deputati, 18 ottobre 2005 (d'ora innanzi Lettera all'on. Casini, 18 ottobre 2005).

${ }_{9}$ Discorso a S.E. Cristina Castañer-Ponce Enrile, Ambasciatore delle Filippine presso la Santa Sede, 27 ottobre 2008.

10 Lettera enciclica "Deus caritas est", 25 dicembre 2005, n. 28.

11 Oriundis e Picena Provincia, Romae degentibus, 25 marzo 1958, in Acta Apostolicae Sedis, 25 (1958), p. 220 (d'ora innanzi Oriundis). 
da Paolo VI'12. E Giovanni Paolo II non ha mancato di ricordare come il cristianesimo abbia riconosciuto "fin dalle sue origini una sana laicità delle strutture della società civile, favorendo la fondamentale distinzione tra l'ordine temporale e l'ordine spirituale"'13.

\section{3 - Laicità e libertà}

È di tutta evidenza che la laicità così descritta ha una stretta connessione con la libertà religiosa, come Benedetto XVI avverte, più che con formulazioni di carattere generale, con la positiva valutazione delle specifiche esperienze di alcuni Paesi, pur tra loro notevolmente diverse. Sotto questo profilo le considerazioni più significative sono proposte in un'intervista concessa ai giornalisti durante uno dei suoi viaggi, dove ha dichiarato: "quanto io trovo affascinante negli Stati Uniti è che hanno incominciato con un concetto positivo di laicità, perché questo nuovo popolo era composto da comunità e persone che erano fuggite dalle Chiese di Stato" e volevano uno Stato laico "proprio per amore della religione nella sua autenticità, che può essere vissuta solo liberamente". Nasce così "uno Stato volutamente e decisamente laico, ma proprio per una volontà religiosa, per dare autenticità alla religione" ${ }^{\prime \prime}$. Considerazioni che, in modo più sintetico, sono riproposte dal pontefice anche a proposito dei regimi vigenti in altri Stati. Così, ad esempio, riguardo alla Turchia, ricorda che nel secolo scorso essa si è dotata

"dei mezzi per divenire un grande Paese moderno, in particolare facendo la scelta di un regime di laicità, distinguendo chiaramente la società civile e la religione, così da permettere a ciascuna di essere autonoma nel proprio ambito, sempre rispettando la libertà dell'altra"'15.

Analogamente, a proposito della laicità francese, il pontefice ritiene che essa "doit aussi permettre de promouvoir davantage l'autonomie de l'Église, que ce soit dans son organisation ou dans sa mission"16.

\footnotetext{
12 Udienza generale, 22 maggio 1968 (d'ora innanzi Udienza generale).

${ }_{13}$ Discorso al IX Colloquio, 11 dicembre 1993, cit, n. 2. Circa tale distinzione vedi anche PONTIFICIO CONSIGLIO DELLA GIUSTIZIA E DELLA PACE, Compendio della dottrina sociale della Chiesa, Libreria Editrice Vaticana, 2004, n. 571, p. 311.

14 Intervista concessa ai giornalisti durante il volo diretto negli Stati Uniti d'America, 15 aprile 2008.

${ }^{15}$ Incontro con il corpo diplomatico presso la Repubblica di Turchia, 28 novembre 2006.

${ }^{16}$ Discours à l'ambassadeur de France, 19 dicembre 2005, cit.
} 
Valutazioni che nel loro complesso meritano particolare attenzione in quanto, in modo ben più esplicito e deciso del precedente magistero, da un lato disapprovano il sistema delle Chiese di Stato e dall'altro riconoscono nella laicità di quest'ultimo la condizione necessaria a una vera libertà religiosa e all'autenticità delle stesse fedi ${ }^{17}$. La loro originalità fa persino apparire non del tutto adeguata la qualifica di "legittima" che i pontefici precedenti avevano talvolta attribuito alla laicità dello Stato $^{18}$, probabilmente allo scopo di giustificarla rispetto alle concezioni tradizionali dello ius publicum ecclesiasticum. Infatti, alla luce delle valutazioni di Benedetto XVI, è da ritenere che la laicità più che lecita, sia necessaria e doverosa, e che, di conseguenza, la Chiesa abbia non solo il compito di rispettarla, ma anche quello di promuoverla. Non sembra dunque un caso che l'attuale pontefice, almeno a quanto risulta, abbia qualificato come "legittima" la laicità dello Stato solo nei primi mesi di pontificato ${ }^{19}$.

\section{4 - Le degenerazioni del principio}

Peraltro Benedetto XVI ha ben presente che esistono altre concezioni della laicità e, in particolare, che "nei tempi moderni" essa ha finito con l'assumere il significato "di esclusione della religione e dei suoi simboli dalla vita pubblica mediante il loro confinamento nell'ambito del privato e della coscienza individuale". Di più: in questo quadro di "crescente emarginazione (...) si tende a considerare la religione, ogni religione, come un fattore senza importanza, estraneo alla società moderna o addirittura destabilizzante" e si cerca di impedirne "ogni forma di rilevanza politica e culturale". Di conseguenza si vuole giungere a una

"totale separazione tra lo Stato e la Chiesa", rifiutando "alla comunità cristiana, e a coloro che legittimamente la rappresentano" il "diritto di pronunziarsi sui problemi morali che oggi interpellano la coscienza di tutti gli esseri umani, in particolare dei legislatori e dei giuristi".

17 Al riguardo merita però ricordare che, secondo Giovanni Paolo II, il riconoscimento della libertà di religione è scaturito dall'atteggiamento assunto dal cristianesimo circa la laicità dello Stato, Discorso al IX colloquio, cit., n. 2.

${ }^{18}$ Vedi Pio XII, Oriundis, cit.; Paolo VI, Udienza generale, cit.

19 Vedi Discorso in occasione della visita al Presidente della Repubblica Italiana S.E. il Signor Carlo Azeglio Ciampi, 24 giugno 2005, e Lettera al Presidente del Senato Marcello Pera in occasione del Convegno di Norcia "Libertà e laicità", 15 ottobre 2005. 
Si intende anche "bandire dalla vita pubblica feste e simboli religiosi in nome del rispetto nei confronti di quanti appartengono ad altre religioni, o di coloro che non credono"20. E, infine, si arriva a "pretendere che i cristiani agiscano nell'esercizio della loro professione senza riferimento alle loro convinzioni religiose e morali, e persino in contraddizione con esse" 21 .

A giudizio del pontefice simili tendenze non sono "espressione di laicità, ma sua degenerazione in laicismo" 22 , in quanto manifestano una "intransigenza secolare (...) nemica della tolleranza"23 e dunque incompatibile con un "corretto concetto" della libertà religiosa ${ }^{24}$. A ben guardare si tratta di un atteggiamento speculare al fondamentalismo, in quanto, al pari di esso, rifiuta il "legittimo pluralismo" e il "principio di laicità"25. In questo modo si genera "una società ingiusta poiché non proporzionata alla vera natura della persona umana", rendendo "impossibile l'affermazione di una pace autentica e duratura di tutta la famiglia umana"26.

20 "Agendo così, non soltanto si limita il diritto dei credenti all'espressione pubblica dello loro fede, ma si tagliano anche radici culturali che alimentano l'identità profonda e la coesione sociale di numerose nazioni".

${ }^{21}$ In particolare "paradossalmente con lo scopo di eliminare le discriminazioni", si ritiene che "i cristiani che rivestano cariche pubbliche dovrebbero, in determinati casi, agire contro la propria coscienza". Per tutti questi insegnamenti vedi, passim, Discorso ai partecipanti al Convegno nazionale promosso dall'Unione Giuristi Cattolici Italiani, 9 dicembre 2006 (d'ora innanzi Discorso all'Unione Giuristi, 9 dicembre 2006); Incontro con le autorità civili durante il viaggio apostolico nel Regno Unito, 17 settembre 2010 (d'ora innanzi Incontro con le autorità del Regno Unito, 17 settembre 2010); Discorso agli eccellentissimi membri del Corpo diplomatico accreditato presso la Santa Sede per la presentazione degli auguri per il nuovo anno, 10 gennaio 2011 (d'ora innanzi Discorso al Corpo diplomatico, 10 gennaio 2011). Si veda anche, più in generale, Incontro con $i$ membri dell' Assemblea generale dell'ONU, 18 aprile 2008 (d'ora innanzi Incontro all'ONU, 18 aprile 2008), dove il pontefice denuncia come "inconcepibile" che, a causa dell"'ideologia secolare prevalente" o delle "posizioni di una maggioranza religiosa di natura esclusiva", i credenti "debbano sopprimere una parte di se stessi - la loro fede - per essere cittadini attivi".

22 Discorso all'Unione Giuristi, 9 dicembre 2006, cit. Si veda anche Discorso ai vescovi della conferenza episcopale di Porto Rico in visita "ad limina Apostolorum", 30 giugno 2007 (d'ora innanzi Discorso alla conferenza di Porto Rico, 30 giugno 2007), n. 5, dove il pontefice lamenta il propagarsi di "un laicismo che, in modo più o meno consapevole, sta gradualmente portando al disprezzo e all'ignoranza del sacro".

23 Discorso ai partecipanti al Convegno promosso dal Partito Popolare Europeo, 30 marzo 2006.

24 Discorso alla conferenza di Porto Rico, 30 giugno 2007, cit., n. 5.

25 Messaggio, $1^{\circ}$ gennaio 2011, cit. n. 8 .

${ }^{26}$ Messaggio, $1^{\circ}$ gennaio 2011, cit., n. 1. 


\section{5 - Laicità sana e laicità positiva}

Dato che le concezioni denunciate sono ampiamente diffuse, il magistero, ogni volta che valuta positivamente la laicità dello Stato, avverte l'esigenza di precisare che non intende riferirsi a qualunque forma di laicità, ma solo a quella che merita la precisa qualifica di "sana", così come era avvenuto nella prima menzione del termine ad opera di Pio XII. Una laicità che, tenendo presente la "duplice dimensione" "religiosa" e "sociale" della persona umana 27 , sancisce "l'effettiva autonomia delle realtà terrene, non certo dall'ordine morale, ma dalla sfera ecclesiastica"28. Una laicità, dunque, che non nega "la dimensione pubblica della religione" 29 sotto il duplice profilo comunitario e personale. Da un lato riconosce la religione, in quanto "organizzata in strutture visibili", come "presenza comunitaria pubblica" 30 e, dall'altro, consente ai credenti di "fare la loro parte nella costruzione dell'ordine sociale" 31 .

A questo proposito merita però segnalare una certa evoluzione nel linguaggio adottato. Sia pure occasionalmente, ma più frequentemente in tempi recenti, Benedetto XVI, nel qualificare la concezione della laicità proposta dalla Chiesa, privilegia l'aggettivo "positiva", rispetto a quello tradizionale di "sana". Il nuovo termine compare per la prima volta il 15 ottobre 2005, nella lettera inviata al Presidente del Senato Marcello Pera, per indicare una laicità "che garantisca ad ogni cittadino il diritto di vivere la propria fede religiosa con autentica libertà anche in ambito pubblico". Riappare poi solamente il 15 aprile 2008 nella intervista concessa ai giornalisti durante il volo diretto negli Stati Uniti. Questa rivisitazione dopo quasi tre anni è probabilmente dovuta al fatto che il presidente della Repubblica francese Sarkozy il 20 dicembre 2007, in occasione dell'accettazione del

27 Messaggio, $1^{\circ}$ gennaio 2011, cit., n. 3.

28 Discorso all'Unione Giuristi, 9 dicembre 2006, cit.

${ }^{29}$ Messaggio, $1^{\circ}$ gennaio 2011, cit., n. 9

30 Discorso all'Unione Giuristi, 9 dicembre 2006, cit. Vedi anche Discorso a S.E. il Signor Charles Ghislain, ambasciatore del Belgio presso la Santa Sede, 24 aprile 2010: la Chiesa "in quanto istituzione ha il diritto di esprimersi pubblicamente. Lo condivide con tutti gli individui e tutte le istituzioni, al fine di dire il suo parere sulle questioni di interesse comune". In altra occasione il pontefice ha sottolineato il "giusto posto che il credo religioso mantiene del dibattito politico", avvertendo che la religione "non è un problema da risolvere, ma un fattore che contribuisce in modo vitale al dibattito pubblico nella nazione": vedi Incontro con le autorità del Regno Unito, 17 settembre 2010, cit.

${ }^{31}$ Incontro all'ONU, 18 aprile 2008 , cit. 
titolo di canonico onorario in San Giovanni in Laterano, aveva testualmente dichiarato, sulla base di ampie e diffuse argomentazioni:

“j'appelle de mes vœux l'avènement d'une laïcité positive, c'est-àdire d'une laïcité, qui tout en veillant à la liberté de pensée, à celle de croire et de ne pas croire, ne considère pas que les religions sont un danger, mais plutôt un atout".

La formulazione di questo auspicio ottenne specifica e puntuale attenzione da parte di Benedetto XVI che il 12 settembre 2008, incontrando le autorità dello Stato francese all'Eliseo, non aveva mancato di ricordare «la bella espressione di "laicità positiva" » usata da Sarkozy.

Dopo un tale apprezzamento sarebbe stato lecito aspettarsi un utilizzo sistematico di questa espressione da parte del magistero, ma l'8 gennaio 2009, nel discorso di Benedetto XVI al Corpo diplomatico accreditato presso la Santa Sede, ricompare la "sana laicità", poi accantonata in favore della "laicità positiva" nel discorso al corpo diplomatico accreditato presso la Santa Sede dell'11 gennaio 201032, come pure nel messaggio per la giornata della Pace del 2011 (n. 9).

Quest'ultima aggettivazione appare, comunque decisamente preferibile in quanto da un lato evita di qualificare senz'altro come "insana" qualunque altra concezione, e dall'altro indica, sia pure implicitamente, trattarsi di una laicità caratterizzata da un atteggiamento aperto nei confronti della religione.

\section{6 - Chiesa e Stato}

Nei discorsi di Benedetto XVI ricorre con una certa frequenza l'affermazione che

"la Chiesa, in Italia e in ogni Paese, come pure nei diversi Consessi internazionali, non intende rivendicare per sé alcun privilegio, ma soltanto avere la possibilità di adempiere la propria missione, nel rispetto della legittima laicità dello Stato" ${ }^{\prime 33}$.

32 Dove si precisa che laicità così qualificata è una laicità "aperta che, fondata su una giusta autonomia tra l'ordine temporale e quello spirituale, favorisca una sana collaborazione e un senso di responsabilità condivisa".

33 Lettera all'on. Casini, 18 ottobre 2005, cit. Vedi anche Discorso ai vescovi della conferenza episcopale di Grecia in visita "ad limina Apostolorum", 30 ottobre 2006 (d'ora innanzi Discorso alla conferenza di Grecia, 30 ottobre 2006); Discorso a S.E. il Signor César Maurizio Velásquez Ossa, nuovo ambasciatore di Colombia presso la Santa Sede, 18 ottobre 2010; Discorso al Corpo diplomatico, 10 gennaio 2011, cit. 
Anche se non viene espressamente citato, in queste enunciazioni è fin troppo trasparente il riferimento a un passo degli insegnamenti conciliari che merita essere riportato integralmente, per sottoporlo poi ad attenta valutazione critica. La costituzione "Gaudium et spes" al n. 76 dichiara: la Chiesa

"si serve di strumenti temporali nella misura in cui la propria missione lo richiede", ma "non pone la sua speranza nei privilegi offerti dall'autorità civile", anzi "rinunzierà all'esercizio di certi diritti legittimamente acquisiti, ove constatasse che il loro uso possa far dubitare della sincerità della sua testimonianza o nuove circostanze esigessero altre disposizioni".

Da più parti, negli anni immediatamente successivi al Concilio, si è ritenuto che queste enunciazioni confermassero la tesi che, nelle relazioni della comunità ecclesiale con la società civile, il Concilio privilegiasse i "rapporti di base" al punto di ripudiare qualunque forma di "rapporti di vertice". In altri termini e ancor più esplicitamente: il Vaticano II sarebbe stato la tomba dei concordati, una previsione smentita dalla esperienza che nei decenni successivi ha visto la stipulazione di numerose conventiones tra la Santa Sede e gli Stati ${ }^{34}$.

In realtà il passo in questione, mentre riconferma la dottrina assolutamente pacifica e del tutto tradizionale che la Chiesa può riporre la propria speranza solo nella "potenza del Signore risorto" 35 , non nega minimamente che essa possa legittimamente acquisire privilegi dall'autorità civile e liberamente servirsene. Avverte soltanto che essa rinuncerà autonomamente al loro esercizio qualora risultassero di obiettivo ostacolo alla propria missione o non rispondessero più alle sue esigenze a causa delle mutate circostanze storiche.

Si può dunque affermare che, in questa materia, le sintetiche e occasionali enunciazioni di Benedetto XVI risultano ben più significative in quanto da un lato non si limitano a prevedere delle eventualità e, dall'altro, non riguardano il passato ma il futuro.

Tuttavia anch'esse non possono leggersi come un ripudio dello strumento concordatario dal momento che questo genere di conventiones ha lo scopo di definire lo status della Chiesa in un determinato Paese, e dunque, soprattutto attualmente, non può

34 Per una esposizione critica di tale tesi vedi G. CASUSCELLI, Concordati, intese e pluralismo confessionale, Milano, Giuffrè, 1974, pp. 82 ss., e M. TEDESCHI, Le attuali relazioni tra Chiesa e Stato, in ID., Saggi di diritto ecclesiastico, Torino, Giappichelli, 1987, pp. 136 ss.

35 Vedi costituzione conciliare "Lumen gentium", n. 8. 
considerarsi genericamente come sinonimo di regime privilegiario. È utile in proposito ricordare che non poche disposizioni degli accordi intervenuti nel 1984 tra la Repubblica italiana e la Santa Sede hanno trovato puntuale riscontro nelle intese intervenute tra la stessa Repubblica e le rappresentanze di altre confessioni religiose.

In ogni caso Benedetto XVI ritiene non solo leciti o opportuni, ma assolutamente indispensabili i "rapporti di vertice", deprecati da taluni autori. Definisce, infatti, "un sano dialogo tra le istituzioni civili e quelle religiose" "fondamentale" "per lo sviluppo integrale della persona umana e dell'armonia della società" 36 . Un dialogo da realizzarsi anche

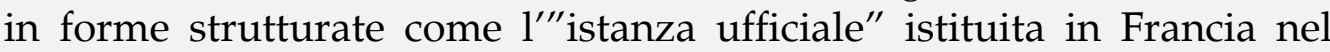
$2002^{37}$, la commissione bilaterale allo studio in Iran "per sviluppare le relazioni e la conoscenza reciproca tra la Repubblica Islamica d'Iran e la Chiesa Cattolica"38, o l'analogo organismo di cui si auspica la creazione in Turchia ${ }^{39}$.

A tali strutture è spesso affidato anche il compito specifico di ottenere da parte dello Stato "il riconoscimento giuridico della Chiesa e dei suoi beni", come si vorrebbe in Turchia, o, più precisamente, la definizione di "uno statuto giuridico" della Chiesa "appropriato e riconosciuto", come avviene in Grecia 40 .

Uno statuto che, come noto, in non pochi Paesi è assicurato dai concordati o da analoghe conventiones, per le quali Benedetto XVI manifesta il più deciso apprezzamento. Afferma, infatti, che tali "patti internazionali" trovano "il loro fondamento nella giusta volontà da parte dello Stato di garantire ai singoli e alla Chiesa il pieno esercizio della libertà religiosa"41. Essi hanno quindi per quest'ultima

"la funzione fondamentale di aiutarla a svolgere la sua missione al servizio di ogni uomo e di tutti gli uomini nella loro vita quotidiana, partecipando così allo sviluppo delle persone e della

\footnotetext{
${ }^{36}$ Messaggio, $1^{\circ}$ gennaio 2011, cit., n. 9.

37 Discorso a Sua Eccellenza il Signor Stanislas Lefebvre de Laboulaye, nuovo ambasciatore di Francia presso la Santa Sede, 26 gennaio 2009 (d'ora innanzi Discorso all'ambasciatore di Francia, 26 gennaio 2009); vedi anche Discorso in occasione dell'incontro con le autorità dello Stato all'Elysée, cit. Per ampie notizie su tale organismo vedi P. VALDRINI, Francia, in AA.VV., Le conferenze episcopali in Europa, a cura di S. Coglievina, Milano, Vita e pensiero, 2010, pp. 3-18.

38 Discorso ai vescovi dell'Iran in visita "ad limina Apostolorum", 16 gennaio 2009.

${ }^{39}$ Discorso ai vescovi della Turchia in visita "ad limina Apostolorum", 2 febbraio 2009.

40 Discorso alla conferenza di Grecia, 30 ottobre 2006.

41 Discorso a S.E. il Signor Francesco Maria Greco, nuovo ambasciatore d'Italia presso la Santa Sede, 17 dicembre 2010.
} 
nazione, e infondendo in ognuno una speranza nuova nel futuro" 42 .

Di conseguenza Benedetto XVI si rallegra che

"Stati di diverse regioni del mondo e di diverse tradizioni religiose, culturali e giuridiche scelgano il mezzo delle convenzioni internazionali per organizzare i rapporti tra la comunità politica e la Chiesa cattolica" 43 .

Convenzioni che - dopo la fioritura ad opera di Giovanni Paolo II - hanno già avuto ulteriore diffusione in questi primi anni del pontificato di Benedetto XVI grazie alla stipulazione di accordi con Paesi che finora non si era avvalsi di questi strumenti o li avevano utilizzati in misura molto parziale. Si vedano, in particolare, il Basic agreement con la Bosnia Erzegovina del 19 aprile 200644; l'accordo con la Repubblica delle Filippine sui beni culturali del 17 aprile 200745; 1'Accord con la Repubblica Francese "separatista" "sur la reconnaissance des grades et diplômes dans l'enseignement supérieur" del 18 dicembre $2008^{46}$ e, di notevole rilevanza, l'Accordo con la Repubblica Federativa del Brasile sullo statuto giuridico della Chiesa Cattolica del 13 novembre $2008{ }^{47}$. Si ha pure notizia che "negoziati sono attualmente in corso in diversi Paesi" 48 .

42 Discorso a S.E. il Signor Firmin Mboutsou, ambasciatore del Gabon presso la Santa Sede, 26 giugno 2008. Si tenga presente che in Gabon vige una convenzione con la Santa Sede per le scuole cattoliche firmata e contestualmente ratificata il 26 luglio 2001, vedi Acta Apostolicae Sedis, 93 (2001), pp. 839-844. Per un commento alla stessa vedi J. MARTíN de AGAR, L'Accordo gabonese sulla scuola cattolica, in Ius Ecclesiae, 14 (2002), pp. 576-581.

43 Discorso al Corpo diplomatico, 10 gennaio 2011, cit.

44 In Acta Apostolicae Sedis, 99 (2007), pp. 939-946.

45 In Acta Apostolicae Sedis, 101 (2009), pp. 1068-1070. Non si conoscono altri accordi della Santa Sede con gli Stati esclusivamente dedicati a questa materia. In precedenza le Filippine avevano sottoscritto una sola conventio riguardante l'erezione del vicariato castrense, si veda il testo del relativo scambio di note in J.T. MARTÍN de AGAR, Raccolta di concordati 1950-1999, Libreria Editrice Vaticana, 2000, pp. 199-204.

46 In Acta Apostolicae Sedis, 101 (2009), pp. 59-64. Il pontefice non ha mancato di dichiararsi "lieto" di questo accordo di cui "beneficeranno numerosi studenti francesi e stranieri", Discorso all'ambasciatore di Francia, 26 gennaio 2009, cit.

47 In Acta Apostolicae Sedis, 102 (2010), pp. 118-129. Per un commento vedi F. VECCHI, Il concordato brasiliano del 2008: dal "separatismo privilegiario" al "pluralismo concordatario" nel modello pattizio di "Vereinbarung", in Ius Ecclesiae, 22 (2010), pp. $427-$ 446.

48 Discorso al Corpo diplomatico, 10 gennaio 2011, cit. Per quanto specificamente riguarda la situazione di Cuba il pontefice auspica che, soprattutto a vantaggio dei cittadini, si possa giungere a "fissare insieme, seguendo forme simili a quelle che si 
Si aggiunga che recentemente "la rete diplomatica della Santa Sede si è ulteriormente consolidata in Africa" e le "Autorità vietnamite hanno accettato" la designazione di "un Rappresentante, che esprimerà nelle sue visite alla (...) comunità cattolica di quel Paese la sollecitudine del successore di Pietro" 49 .

\section{7 - Riflessioni conclusive}

In tutti questi insegnamenti Benedetto XVI non intende certo enunciare nuovi principi in materia di libertà religiosa e di rapporti della Chiesa con lo Stato. Vuole solo riflettere sulla dottrina tradizionale così come rivisitata dai suoi immediati predecessori, ma non si limita a ripetere quanto già acquisito, attualizzandolo e reinterpretandolo secondo la propria sensibilità e le specifiche esigenze dei tempi.

Sotto questo profilo è indubbiamente significativo che la sua riflessione in materia si polarizzi in larga misura intorno alla problematica della laicità dello Stato: una questione certo ben presente ai suoi immediati predecessori, ma senza una tale rilevanza.

Come si è visto, Benedetto XVI continua a riconoscere in tale laicità un principio cristiano in quanto, in ultima analisi, essa consiste in quella distinzione dei poteri che è stata sancita da Cristo stesso nel ben noto episodio evangelico del tributo a Cesare, ed è stata quindi proposta dalla Chiesa fin dalle origini. Ma, al tempo stesso, l'attuale pontefice offre una lettura non priva di originalità di questa dottrina del tutto tradizionale.

In proposito è opportuno ricordare che la distinzione in questione è così presentata dal Concilio: "La comunità politica e la Chiesa sono indipendenti e autonome l'una dall'altra nel proprio campo $^{\prime \prime 50}$. Una formulazione che ricalca fedelmente quella adottata da Leone XIII nella enciclica "Immortale Dei" 51: "Dio volle ripartito tra due poteri il governo del genere umano, cioè il potere ecclesiastico e quello

stabiliscono con altre Nazioni e rispettando le caratteristiche proprie del (...) Paese, un quadro giuridico che definisca convenientemente le relazioni esistenti e mai interrotte" con la Santa Sede e "garantisca un adeguato sviluppo della vita e dell'azione pastorale della Chiesa", Discorso a S.E. il Signor Eduardo Delgado Bermúdez, ambasciatore di Cuba presso la Santa Sede, 10 dicembre 2009.

49 Discorso al Corpo diplomatico, 10 gennaio 2011, cit. Per quanto specificamente riguarda degli Emirati Arabi Uniti, vedi il Discorso a S.E. la Signora Hissa Abdulla Ahmed Al-Otaiba, primo ambasciatore degli Emirati Arabi Uniti presso la Santa Sede, 20 maggio 2010.

50 Costituzione conciliare "Gaudium et spes", n. 76.

51 De civitatum constitutione christiana, $1^{\circ}$ novembre 1885. 
civile, l'uno preposto alle cose divine, l'altro alle umane. Entrambi sono sovrani nella propria sfera".

Dagli stessi termini utilizzati dal pontefice risulta evidente che l'affermazione di questo principio ha come funzione principale ed essenziale quella di rivendicare l'indipendenza della Chiesa di fronte a qualunque potere civile. Una indipendenza, che è bene ricordare, il Codice di diritto canonico ha cura di sancire espressamente in diverse norme riguardanti specifiche materie ${ }^{52}$.

Ovviamente Benedetto XVI non nega tutto questo, ma nel suo insegnamento, almeno finora, privilegia una diversa prospettiva. Infatti, come si è avuto modo di mettere in luce, da un lato fonda la laicità dello Stato sulla distinzione dei poteri finendo quasi con l'identificarla con la stessa, ma dall'altro propone una concezione della laicità "sana", o meglio "positiva", che lasci cioè doveroso spazio al contributo che la Chiesa può offrire alla costruzione della società degli uomini in questo mondo. In altri termini: più che dell'indipendenza della Chiesa dallo Stato, si preoccupa, di fronte ai molteplici tentativi di emarginazione, della presenza della Chiesa nelle contingenze politiche, sociali ed economiche.

Una preoccupazione che concerne non solo la Chiesa, ma, più ampiamente, la religione nella sua dimensione personale e comunitaria. E per quanto specificamente riguarda le confessioni religiose la legittimità della loro presenza nello spazio pubblico non è giustificata con le prerogative proprie delle stesse ${ }^{53}$, ma con il diritto di qualunque soggetto, individuale o comunitario, ad esprimersi liberamente in ambito sociale e a partecipare attivamente al dibattito politico.

Peraltro l'attenzione di Benedetto XVI per i profili che concernono specificamente la libertas Ecclesiae ha modo di esprimersi sia con affermazioni di portata generale presenti in alcuni discorsi, sia, soprattutto, nella concretezza dell'azione diplomatica. Infatti egli, come si è avuto modo di evidenziare, mentre ripudia le Chiese di Stato, si preoccupa che la comunità ecclesiale abbia nei diversi Paesi un statuto giuridico adeguato e appropriato ${ }^{54}$. E per quanto riguarda la concreta definizione dello stesso mostra di apprezzare, per non dire preferire, lo strumento dei concordati o di simili conventiones, che già nella loro

\footnotetext{
52 Se ne veda una rassegna in G. FELICIANI, La Chiesa di fronte agli Stati, in La Scuola Cattolica, 124 (1996), pp. 264-266 e 281-282.

53 Enunciate da Giovanni Paolo II nel Messaggio ai Capi di Stato dei Paesi firmatari dell'atto finale di Helsinki, $1^{\circ}$ settembre 1980, n. 4.

54 Da segnalare anche, soprattutto in questi ultimi tempi, la sua preoccupazione per le persecuzioni di cui sono oggetto i cristiani in vari Paesi del mondo, vedi, ad es., Messaggio, $1^{\circ}$ gennaio 2011, cit., n. 1 .
} 
stipulazione comportano il riconoscimento da parte degli Stati interessati della indipendenza e della sovranità della Chiesa negli ambiti che le sono propri. 